\title{
Oral Cancer Diagnostics using FTIR-ATR Spectra of Salivary Exosomes: A Preliminary Study
}

\author{
B.Z. Dekel ${ }^{1}$, A. Zlotogorski-Hurvitz ${ }^{2,3}$, D. Malonek ${ }^{1}$, R. Yahalom ${ }^{4}$, M. Vered ${ }^{2,5}$ \\ ${ }^{1}$ Dept. of Electrical \& Computer Engineering, Ruppin Academic Center \\ Emek Hefer, Israel. \\ benziond @ ruppin.ac.il \\ ${ }^{2}$ Dept. of Oral Pathology \& Oral Medicine, School of Dentistry, Tel-Aviv University \\ Tel Aviv, Israel. \\ ${ }^{3}$ Dept. Oral \& Maxillofacial Surgery, Rabin Medical Center \\ Petah Tikva, Israel. \\ ${ }^{4}$ Dept. Oral \& Maxillofacial Surgery, The Chaim Sheba Medical Center, Tel Hashomer \\ Ramat Gan, Israel. \\ ${ }^{5}$ Institute of Pathology, The Chaim Sheba Medical Center, Tel Hashomer \\ Ramat Gan, Israel.
}

\section{Extended Abstract}

Oral cancer (OC), referring to the main variant of squamous cell carcinoma, is now assessed to have a global incidence of over 300,000 new annual cases with a trend to further increase in younger patients and in developing countries. One of the caveats associated with OC is the ability to identify early changes in the oral lining epithelium mainly in those patients at high risk to develop oral cancer.

Tissue biopsy and light microscopy are still the gold-standard diagnostic tool. However, these are performed when lesions (cancerous or pre-cancerous) are visible and already contain substantial genetic changes. Moreover, tissue biopsies provide information that is limited to a portion of a tumor and at a specific time-point. Liquid biopsy, based on different body fluids, is currently a new concept in cancer diagnostics as it can provide information at successive time points on circulating tumor cells. In this line, saliva can be used as a promising biofluid for the early identification of biomarkers for both local and systemic diseases. In our research salivary extracellular microvesicles, like exosomes, have been isolated from oral cancer patients and were shown to demonstrate different mid infrared spectrum from those of healthy individuals.

Our aim was to use FTIR spectroscopy combined with machine learning methods for the primary evaluation of the characteristic spectra of salivary exosomes from OC patients and healthy individuals (HI), thus creating a novel platform for the application of FTIR spectroscopy for the early diagnosis of oral cancer.

The study [1] was approved by relevant ethics committees and was conducted in accordance with the Declaration of Helsinki. Whole saliva samples were collected from 21 oral cancer patients and 13 healthy. Exosomes were pelleted using differential centrifugation $(12,000 \mathrm{~g}, 120,000 \mathrm{~g})$. Following the mid-IR absorbance spectra $\left(900 \mathrm{~cm}^{-1}-5,000 \mathrm{~cm}^{-1}\right.$ range) was measured using an ATR-FTIR. Machine learning techniques, utilized to build discrimination models for the absorbance data of the measured samples, included the (PCA-LDA) and (SVM) classification. Sensitivity and specificity were calculated.

Our discrimination model was able to differentiate IR spectra of oral cancer from healthy using absorption bands at $1,072 \mathrm{~cm}^{-1}$ (nucleic acids), 2,924 $\mathrm{cm}^{-1}$ and $2,854 \mathrm{~cm}^{-1}$ (membranous lipids) and $1,543 \mathrm{~cm}^{-1}$ (transmembrane proteins). The PCALDA discrimination model correctly classified the samples with a sensitivity of $100 \%$, specificity of $89 \%$ and accuracy of 95\% and the SVM showed a training accuracy of $100 \%$ and a cross validation accuracy of $89 \%$.

Even though the population of exosomes in saliva of OC patients is an admixture of tumor cells and the non-cancerous oral epithelium, the discriminant model could distinguish with high accuracy the spectra of OC exosomes from those of $\mathrm{HI}$ exosomes, indicating that the exclusive features of the cancerous exosomes prevailed.

The present findings are important as they show that cancer exosomes can be accurately differentiated from their benign counterparts using machine learning techniques with small data sets for processing the spectral data. This may have an important role for the development of next-generation techniques for the early diagnosis of oral cancer. 


\section{References}

[1] A. Zlotogorski-Hurvitz, B. Z. Dekel, D. Malonek, et al. "FTIR-based spectrum of salivary exosomes coupled with computational-aided discriminating analysis in the diagnosis of oral cancer," J Cancer Res Clin Oncol, 2019. 